\title{
Myeloid sarcoma derived from the gastrointestinal tract: A case report and review of the literature
}

\author{
TENG YU, GENBO XU, XIAOHUA XU, JING YANG and LUYIN DING \\ Department of Hematology, The Second Affiliated Hospital of Zhejiang University School of Medicine, \\ Hangzhou, Zhejiang 310009, P.R. China
}

Received March 23, 2015; Accepted April 12, 2016

DOI: $10.3892 / 01.2016 .4517$

\begin{abstract}
Myeloid sarcoma is a type of malignant neoplasm composed of myeloblasts that locates extramedullary. The present study reports the case of a 31-year-old female who presented with upper abdominal pain, melena, vomiting and jaundice. The abdominal computed tomography revealed a mass in gastric antrum area and possible infiltration of the duodenum, gallbladder and head of the pancreas, with possible retroperitoneal lymph node metastasis. The tumor grew quickly and led to serious obstructive jaundice. New masses developed in the bilateral orbits and left breast within 2 months of admission. The pathological results of the gastroscopic biopsy and the fine-needle biopsy of the breast revealed myeloid sarcoma. Transhepatic cholangial drainage, radiotherapy and chemotherapy were administered, but the disease reoccurred and became resistant to chemotherapy, so salvage allogenetic peripheral blood stem cell transplantation was performed. The disease relapsed at 5 months post-transplantation, and chemotherapy and donor lymphocytes transfusions were then administered. The patient declined further treatment and succumbed to disease on May 19, 2015. The present study could improve the understanding of myeloid sarcoma and provide a reference for standardized and individualized treatments for this disease.
\end{abstract}

\section{Introduction}

Myeloid sarcoma is a malignant neoplasm of myeloid origin that locates outside the bone marrow. The tumor is also known as a chloroma due to its green color when exposed to air. Myeloid sarcoma can develop in any area of the body, including the cervix, mediastinum, small intestines, lymph nodes and skin (1). In the majority of cases, myeloid sarcoma

Correspondence to: Dr Luyin Ding, Department of Hematology, The Second Affiliated Hospital of Zhejiang University School of Medicine, 88 Jiefang Road, Hangzhou, Zhejiang 310009, P.R. China E-mail: dingly85@163.com

Key words: myeloid sarcoma, granulocytic sarcoma, chloroma, gastrointestinal tract, myeloperoxidase is an extramedullary presentation of leukemia, and in a few cases, it can develop prior to onset or following the remission of leukemia, presenting as a solitary extramedullary neoplasm known as a solitary myeloid sarcoma or primary myeloid sarcoma (2). The incidence of myeloid sarcoma is only $1-2 \%$ of all acute myeloid leukemia cases, and only $6.5 \%$ of myeloid sarcomas derive from the gastrointestinal tract (3). These patients usually present with nonspecific symptoms, including abdominal pain, nausea, vomiting, bowel obstruction or gastrointestinal bleeding without bone marrow involvement, thus being easily misdiagnosed (4). The present study reports a case of myeloid sarcoma derived from the gastrointestinal tract and reviews the associated literature, in order to improve the understanding of the disease and provide a reference for standardized and individualized treatments.

\section{Case report}

A 31-year-old female was admitted to the Second Affiliated Hospital of Zhejiang University School of Medicine (Hangzhou, Zhejiang, China) on June 20, 2012, due to upper abdominal pain and melena that had been apparent for 2 months and vomiting that had persisted for 1 month. Physical examinations showed jaundice of the skin and sclera, no compression pain of the sternum and no enlarged superficial lymph nodes. The patient's abdomen was soft, with moderate tenderness, but with no rebound pain and no palpable mass. The liver and spleen were not palpable beneath the ribs. A routine blood test showed a white blood cell count of $9.5 \times 10^{9}$ cells/1 (normal range, $4.0-10.0 \times 10^{9}$ cells/l), a hemoglobin level of $138 \mathrm{~g} / 1$ (normal range, $110-160 \mathrm{~g} / \mathrm{l}$ ) and a platelet level of $202 \times 10^{9}$ cells $/ 1$ (normal range, $100-300 \times 10^{9}$ cells/l). The abdominal enhanced computed tomography (CT) scan (Somatom Definition AS 64-row CT scanner; Siemens AG, Munich, Germany) revealed a mass in the gastric antrum area, $5.2 \times 6.2 \mathrm{~cm}$ in size, with possible infiltration of the duodenum, gallbladder and head of the pancreas, and possible retroperitoneal lymph node metastasis (Fig. 1). Gastroscopy revealed chronic gastritis and a mass in the cavity of the duodenal bulb, from which a biopsy sample was taken. Positron emission tomography-CT revealed a high metabolic tumor in the area of the duodenal bulb. The tumor was $5.2 \times 6.2 \mathrm{~cm}$ in size and its maximum standard uptake value (SUVmax) was 10.39. The tumor was adhered to the head of the pancreas, the gastric antrum and 


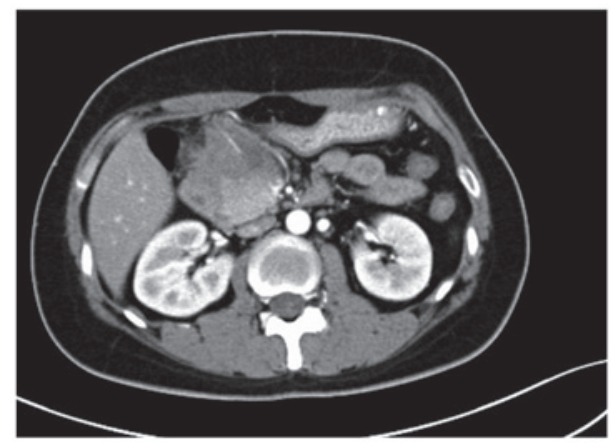

Figure 1. Abdominal computed tomography enhanced scan on June 22, 2012, revealing a mass in the gastric antrum area, $5.2 \times 6.2 \mathrm{~cm}$ in size, with possible infiltration of the duodenum, gallbladder and head of the pancreas, and possible retroperitoneal lymph node metastasis.

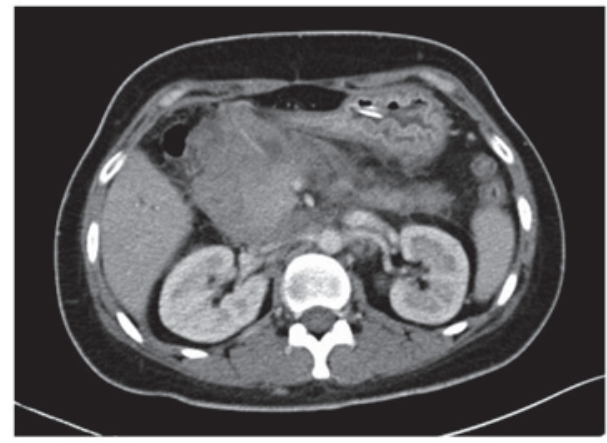

Figure 2. Abdominal enhanced computed tomography scan on July 14, 2012, showing marked enlargement of the tumor $(12 \times 9 \mathrm{~cm})$, and expansion of the superior segment of the common bile duct and the intrahepatic bile ducts.

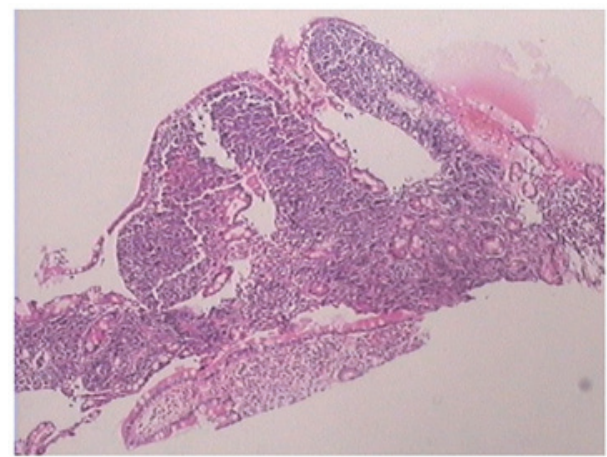

Figure 3. Pathological findings of the gastroscopic biopsy showing large abnormal cells with loose chromatin and nucleoli (hematoxylin-eosin staining; magnification, x100). The diagnosis was confirmed as myeloid sarcoma according to immunohistochemical staining.

the liver. Multiple hypermetabolic lymph nodes were found in the retroperitoneal area with an SUVmax of 5.03. Repeat abdominal CT after 9 days showed marked enlargement of the tumor $(12 \times 9 \mathrm{~cm})$, and expansion of the superior segment of the common bile duct and the intrahepatic bile ducts. This was considered to be due to compression of the bile duct (Fig. 2). The jaundice and liver function of the patient worsened, with test results as follows: Total bilirubin, $180.4 \mu \mathrm{mol} / 1$ (normal range, 3.4-20.5 $\mu \mathrm{mol} / \mathrm{l}$ ); direct bilirubin, $147.0 \mu \mathrm{mol} / 1$ (normal range, $<6.8 \mu \mathrm{mol} / \mathrm{l}$ ); alanine aminotransferase, $761 \mathrm{U} / 1$ (normal

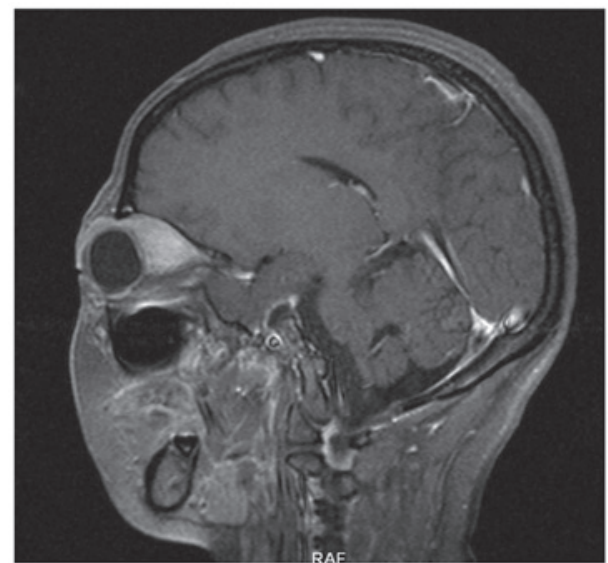

Figure 4. Magnetic resonance imaging of the orbits on July 25, 2012, showing tumors in the bilateral superior rectus and eyelids.

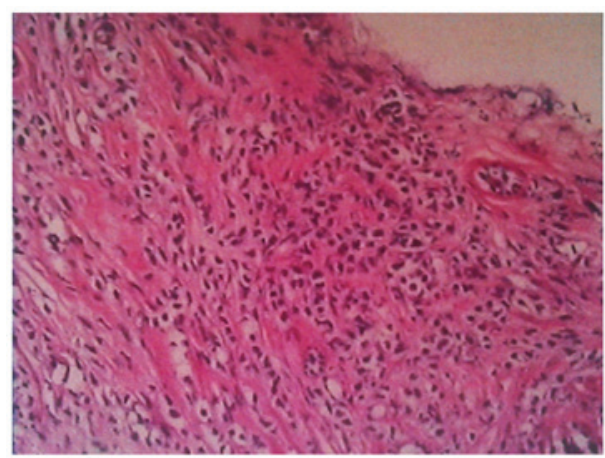

Figure 5. B-mode ultrasound-guided fine-needle biopsy of the breast mass showing fibrous tissues infiltrated by abnormal cells (hematoxylin-eosin staining; magnification, x100). Immunohistochemical staining revealed myeloid sarcoma.

range, <34 U/l); aspartate aminotransferase, $596 \mathrm{U} / 1$ (normal range, $<35 \mathrm{U} / \mathrm{l}$ ); alkaline phosphatase, 1,092 U/1 (normal range, 30-120 U/1); $\gamma$-glutamyl transpeptidase, $1451 \mathrm{U} / 1$ (normal range, 9-64 U/1); and lactate dehydrogenase, $489 \mathrm{U} / 1$ (normal range, 140-271 U/1). The pathological results of the gastroscopic biopsy showed large abnormal cells with loose chromatin and nucleoli, and immunohistochemical staining revealed that the tumor cells expressed cluster of differentiation (CD)43 and myeloperoxidase (MPO), with scattered CD123 and terminal deoxynucleotidyl transferase expression (Fig. 3). The diagnosis was of myeloid sarcoma. Bone marrow routine tests and immunophenotyping were normal, however, gene screening of the bone marrow cells showed increased MDS1 and EVI1 complex locus (EVI1) gene expression. A percutaneous transhepatic cholangial drainage (PTCD) procedure was performed on July 17, 2012, in order to relieve the symptom of jaundice, and $300 \mathrm{ml}$ of yellow-green colored bile was drained out everyday. Subsequent to PTCD, the bilirubin level gradually decreased, liver function improved and the jaundice gradually disappeared. Radiotherapy $(6,000,000 \mathrm{eV}$ X-ray; total dose, 2,450 cGy; Clinac 21EX; Varian Medical Systems, Inc., Palo Alto, CA, USA) was administered to the abdominal tumor 7 times (350 cGy each) after July 25, 2012, and the tumor gradually became smaller. However, masses later appeared in the bilateral orbits almost at the beginning 
of radiotherapy, and the mass in the left orbit became progressively enlarged in the subsequent days. Simultaneously a mass was palpable in the left breast. Magnetic resonance imaging (Magnetom Sonata 3.0T; Siemens AG) of the orbits showed tumors in the bilateral superior rectus and eyelids (Fig. 4). B-mode ultrasound showed a hypoechoic area in the left breast. B-mode ultrasound-guided fine-needle biopsy of the breast mass revealed fibrous tissues infiltrated by abnormal cells and immunohistochemical staining revealed a myeloid sarcoma.

Immunohistochemistry was performed with the HistostainPlus IHC kit (Dako, Glostrup, Denmark). Briefly, tumor tissue samples, which were preserved in $2.5 \%$ glutaraldehydepolyoxymethylene solution, were dehydrated and embedded in paraffin following routine methods. For immunohistochemistry, paraffin was removed, and the sections were immersed in distilled water, prior to be rinsed 3 times for 5 min each in $0.01 \mathrm{M}$ phosphate-buffered saline (PBS; pH 7.4) containing $0.05 \%$ Tween 20 (PBST; Fresenius Kabi, Bad Homburg vor der Höhe, Germany), and then blocked with $3 \%$ peroxidemethanol at room temperature for endogenous peroxidase ablation. All the subsequent steps were conducted in a moist chamber: Sections were incubated with blocking buffer (normal goat serum) at room temperature for $20 \mathrm{~min}$, followed by incubation for $2 \mathrm{~h}$ at $37^{\circ} \mathrm{C}$ with mouse anti-MPO, anti-cytokeratin AE1/AE3, anti-epithelial membrane antigen (EMA), anti-vimentin, anti-CD45, anti-Ki-67, anti-CD20, anti-CD79a, anti-CD10, anti-CD3, anti-CD45RO, anti-CD117, anti-CD15 and anti-CD43 antibodies. Upon rinsing in PBST ( $3 \times 5 \mathrm{~min})$, sections were incubated for $30 \mathrm{~min}$ at $37^{\circ} \mathrm{C}$ with goat antimouse immunoglobulin G. Next, sections were rinsed in PBST ( $3 \times 5 \mathrm{~min})$ and incubated with streptavidin-horseradish peroxidase conjugate at $37^{\circ} \mathrm{C}$ for $30 \mathrm{~min}$. Following rinsing in PBST (3x5 min), sections were stained with 3,3'-diaminobenzidine at room temperature for $10 \mathrm{~min}$. Subsequently, sections were washed with distilled water and stained with hematoxylin, prior to dehydration, clearing and mounting with neutral gums. The negative control was analyzed as above, with the exception of the replacement of the aforementioned antibodies by PBS. The immunohistochemical results were as follows: Cytokeratin AE1/AE3(-), EMA(-), vimentin(-), CD45 (leukocyte common antigen)(++), MPO(+++), Ki-67 of $70 \%(+)$, CD20(-), CD79a(-), CD10(-), CD3(-), CD45RO(-), CD117(++), CD15(+) and CD43(++) (Fig. 5).

Myeloid sarcoma involving the breast and orbits was considered. An idarubicin and cytarabine regimen (10 $\mathrm{mg}$ idarubicin on days 1-3 and $150 \mathrm{mg}$ cytarabine on days 1-7) was administered between August 3 and August 9, 2012, after which, the tumors in the gastrointestinal tract, orbits and breast decreased in size. Radiotherapy and consolidation chemotherapy were administered at regular intervals, as follows: Two additional cycles of idarubicin and cytarabine regimens $(10 \mathrm{mg}$ idarubicin on days 1-3, and $150 \mathrm{mg}$ cytarabine on days 1-7) were administered on September 13 and October 30, 2012; a medium dose of cytarabine ( $2 \mathrm{~g}$ cytarabine every $12 \mathrm{~h}$ on days 1-3) was administered on December 14, 2012; and FLAG regimen [40 mg fludarabine on days 1-5, $2.8 \mathrm{~g}$ cytarabine on days $1-5$ and $200 \mu \mathrm{g}$ granulocyte-colony stimulating factor (G-CSF) on days 0-5] was administered on March 1, 2013. However, in April 2013, the tumor in the breast progressed and became resistant to chemotherapy, therefore, salvage allogeneic peripheral blood stem cell transplantation was performed on May 6, 2013. The peripheral hematopoietic stem cells were obtained from an unrelated donor of the China Marrow Donor Program (http://www.cmdp.org.cn/show/1022765.html), and 9 of 10 human leukocyte antigens were matched. The volume of the graft was $191 \mathrm{ml}$, in which the number of mononuclear cells was $5.42 \times 10^{8}$ cells $/ \mathrm{kg}$, and the number of CD34-positive cells was $4.06 \times 10^{6}$ cells $/ \mathrm{kg}$. Consolidation radiotherapy (6,000,000 eV X-ray; total dose, 2,400 cGy) was administered to the orbit tumor 12 times (200 cGy each) after June 11, 2013.. At 5 months post-transplantation, a routine B-mode ultrasound examination found a hypoechoic, $7.1 \times 5.1-\mathrm{cm}$ mass on the top left side of the uterus, which suggested disease relapse. In consequence, the patient received $\mathrm{HAG}$ regimen chemotherapy (1 mg homoharringtonine on days 1-14, $50 \mathrm{mg}$ cytarabine on days 1-14 and $300 \mu \mathrm{g}$ G-CSF on days $0-14$ ) on November 13, 2013. Donor lymphocytes infusions were performed on December 9, 2013 (total mononuclear cells, 3.42 $\times 10^{7}$ cells/

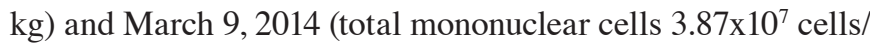
$\mathrm{kg}$ ). The patient declined further treatment and succumbed to disease at home on May 19, 2015.

\section{Discussion}

The primary symptoms of the patient in the present study were bleeding and obstruction of the gastrointestinal tract caused by the tumor. After initial relief from the symptoms, tumors in the orbits and left breast were found. The pathological results of the tumors in the abdomen and left breast confirmed a diagnosis of myeloid sarcoma. Similar cases have seldom been reported globally.

Myeloid sarcoma can be found in patients with a wide spectrum of ages, with cases reported in individuals between 5 months and 89 years old. Despite this, the tumor mainly affects children and the younger population, with no clear gender preference (2). Myeloid sarcoma turns green when exposed to air, so it is also known as chloroma. This change occurs due to the presence of peroxidase, however, not all myeloid sarcomas are green and certain tumors are white in color. The nomenclature of such tumors at present is myeloid sarcoma or granulocytic sarcoma (1). There are four clinical presentations of myeloid sarcoma (5): i) Accompanied with acute myeloid leukemia (AML); ii) could be the portent of AML; iii) could be associated with myelodysplastic disease transforming to leukemia; and iv) solitary tumors. However, such localized primitive granulocytic sarcomas are extremely rare and they could develop to AML if no treatment is provided (5). The case in the current study presented as multiple solitary tumors and the routine bone marrow examination was normal. However, EVI1 gene overexpression occurred in the bone marrow cells, which suggested a poor prognosis (6). Therefore, the standard chemotherapy and radiotherapy were followed by an allogenic peripheral blood stem cell transplantation.

Myeloid sarcoma can be divided into three pathological categories according to the proportion of immature granulocytes in different stages of differentiation: The blast cell type, the partially-differentiated type and the differentiated type (7). Blast cell-type myeloid sarcoma mainly consists of myeloblasts, and there are few differentiated promyelocytes. 
The partially-differentiated type mainly consists of myeloblasts and promyelocytes. The differentiated type mainly consists of promyelocytes and granulocytes of late mature stages, which mostly include eosinophilic granulocytes. The diagnosis is often difficult according to routine histology slices, as the tumor consists of relatively consistent immature cells, particularly in the following situations: i) When the tumor is solitary and no leukemia is evident in peripheral blood smears or bone marrow smears; ii) when the tumor is located in less common areas, such as the small intestines; iii) when the tumor is not green; and iv) when the tumor cells are poorly differentiated (4)

MPO has a high sensitivity and specificity for myeloid cells, and its expression rate in myeloid tumors is as high as 93\%, so it is the most important marker for myeloid sarcomas. However, Mourad et al (8) reported that only $66 \%$ cases of myeloid sarcomas express MPO. Traweek et al (9) reported that MPO is the most useful marker for distinguishing myeloid sarcomas, but that the expression of MPO varies with degrees of differentiation. Using MPO accompanied with CD68, CD43 and CD20,>96\% of myeloid sarcomas could be distinguished. Furthermore, the lysozyme expression rate is $60-93 \%$ in myeloid sarcomas, which could be due to expression in granulocytes and monocytes. However, the specificity of lysozyme is less than that of MPO, as a lot of tissues contain lysozyme. Other studies (10) have suggested that lysozyme and its relative antigen, CD68, are the most sensitive markers for myeloid cells, which makes them of great significance for the diagnosis of myeloid sarcomas. A study by Amador-Ortiz et al (11) analyzed 82 cases of myeloid sarcoma and found that the expression rate of MPO and lysozyme were 95.9 and $95.5 \%$, respectively. CD43 is expressed in almost all myeloid sarcomas, but it is often used to mark T cells, so it has a high sensitivity but poor specificity. When tumor cells of unknown origin express CD43 but are negative for CD3, the possibility of myeloid sarcoma should be considered. CD68 and CD117 are sensitive markers of myeloid tumors that are mainly expressed in immature myeloid tumors and are not expressed in lymphomas. The use of two such markers is extremely useful in distinguishing between myeloid sarcoma and lymphomas. CD45 exhibits moderate positive expression in myeloid sarcomas. CD20 is a characteristic differentiation antigen of B cells, and it is expressed by B cells from the pre-B cell period until their differentiation into plasma cells (10). The majority of studies consider that myeloid sarcomas are CD20-negative, however, Mourad et al (8) reported that the CD20 expression rate is $13 \%$ in myeloid sarcomas.

The literature has reported varied cytogenetic abnormities in myeloid sarcomas (12), such as the inversion of chromosome 16 and its associated molecular genetic change resulting in the core binding factor $\beta /$ myosin, heavy chain 11 , smooth muscle (CBF $\beta /$ MYH11) fusion gene (13). In the present case, the patient exhibited a normal karyotype of 46,XX and was negative for the $\mathrm{CBF} \beta / \mathrm{MYH} 11$ fusion gene; however, EVI1 gene overexpression was recorded.

The differential diagnosis of myeloid sarcoma should include several diseases (14): i) Non-Hodgkin's lymphoma (NHL). No eosinophilic granulocytes are present in NHL tissues, and NHL can express differentiation antigens of B or T lymphocytes instead of MPO or lysozyme. ii) Ewing's sarcoma or primitive neuroectodermal tumors. These diseases are mostly observed in younger individuals. Real or false rosettes can be found without expression of hematological markers such as CD45 or MPO. iii) High risk small cells type stromal tumors. The mesenchymomas usually have clear boundaries to the naked eye and certain tumors exhibit pseudocapsules. Under the microscope, fusiform cells and epithelioid differentiated areas can be found, as well as tumor cells without acidophile cell plasma. The immunophenotyping is positive for CD117 and discovered on GIST-1, but negative for MPO and CD45. Myeloid sarcomas have the appearance of lymphomas and exhibit infiltrative growth without clear boundaries. Under the microscope, similar diffuse small cells without fusiform areas are present, with almost no interstitial elements. Occasionally areas of fibrosis can be found. Tumor cells with acidophile cell plasma may be found, and immunophenotyping reveals positive expression for CD117, MPO, B-cell lymphoma 2 and CD43. iv) Undifferentiated small cell lung cancer. The cancer cells are diffusely distribute, but with a tendency for nest forming. Mitotic figures can be easily observed. EMA and PCK are positively expressed, but the markers for myeloid tissues are negative. In conclusion, the correct diagnosis of myeloid sarcoma could be made with carefully observation of hematoxylin-eosin staining slides, observation of tumor cells differentiating to granulocytes in mature stages, and following integration of these findings with immunophenotypical staining (15).

The prognosis of patients with myeloid sarcoma is extremely poor and the majority succumb to the disease within a short time. Few patients experience long complete remission periods after effective treatments. Untreated primary myeloid sarcoma ultimately transforms to AML usually within 10 months of the diagnosis of myeloid sarcoma. However, in rare instances, cases have been reported in which transformation to leukemia has not occurred in a follow-up time of $>16$ years (16). Yamauchi and Yasuda (17) reported that the median time for myeloid sarcoma transformation to AML is $10-12$ months.

Previously, treatments for primary myeloid sarcoma have included surgical resection, local radiotherapy and systemic chemotherapy. However, surgical resection and local radiotherapy cannot delay the transformation from myeloid sarcoma to AML or improve the prognosis (18). This indicates that primary myeloid sarcoma is a type of systemic disease that consequently requires systemic treatment. Systemic chemotherapy should perform for all solitary myeloid sarcomas, even if surgical resections have been performed. The present hypothesis is that the application of anti-leukemia chemotherapy soon after surgery is useful for controlling the develop of the disease and improving the prognosis (19). The preferred regimen uses anthracyclines combined with cytarabine (20). Allogeneic hematopoietic stem cell transplantation could also be an effective treatment for myeloid sarcoma $(21,22)$. Yagi et al (23) reported the successful treatment of a case of myeloid sarcoma using allogeneic hematopoietic stem cell transplantation following systemic chemotherapy and additional radiotherapy.

In conclusion, myeloid sarcoma is a malignant neoplasm of myeloid origin that could develop in any area of the body. Myeloid sarcoma derived from the gastrointestinal tract 
is relatively rare and tends to be misdiagnosed. Intensive systemic chemotherapy and allogeneic hematopoietic stem cell transplantation should be performed in addition to surgical resection and radiotherapy. The prognosis of myeloid sarcoma remains poor, and further randomized controlled trials are required to improve clinical practice

\section{References}

1. Vardiman JW: The World Health Organization (WHO) classification of tumors of the hematopoietic and lymphoid tissues: An overview with emphasis on the myeloid neoplasms. Chem Biol Interact 184: 16-20, 2010.

2. Pantanowitz L and Thompson L: Myeloid sarcoma. Ear Nose Throat J 84: 470-471, 2005.

3. Pileri SA, Ascani S, Cox MC, Campidelli C, Bacci F, Piccioli M, Piccaluga PP, Agostinelli C, Asioli S, Novero D, et al: Myeloid sarcoma: Clinico-pathologic, phenotypic and cytogenetic analysis of 92 adult patients. Leukemia 21: 340-350, 2007.

4. Narayan P, Murthy V, Su M, Woel R, Grossman IR and Chamberlain RS: Primary myeloid sarcoma masquerading as an obstructing duodenal carcinoma. Case Rep Hematol 2012: 490438, 2012.

5. Shimizu H, Saitoh T, Hatsumi N, Takada S, Yokohama A, Handa H, Jimbo T, Sakura T, Tsukamoto N, Murakami H, et al: Clinical significance of granulocytic sarcoma in adult patients with acute myeloid leukemia. Cancer Sci 103: 1513-1517, 2012.

6. Haferlach C, Bacher U, Grossmann V, Schindela S, Zenger M, Kohlmann A, Kern W, Haferlach T and Schnittger S: Three novel cytogenetically cryptic EVI1 rearrangements associated with increased EVI1 expression and poor prognosis identified in 27 acute myeloid leukemia cases. Genes Chromosomes Cancer 51: 1079-1085, 2012.

7. Li JM, Liu WP, Zhang MH, Wei X, Gu JM, Han AJ, Wu WQ and Chen XY: Clinicopathologic and immunophenotypic analysis of myeloid sarcoma. Zhonghua Bing Li Xue Za Zhi 35: 606-611, 2006 (In Chinese).

8. Mourad W, Kfoury H and Al Husseini H: The value of CD34, myeloperoxidase and chloroacetate esterase (Leder) stain in the diagnosis of granulocytic sarcoma. Ann Saudi Med 21: 287-291, 2001.

9. Traweek ST, Arber DA, Rappaport H and Brynes RK: Extramedullary myeloid cell tumors. An immunohistochemical and morphologic study of 28 cases. Am J Surg Pathol 17: 1011-1019, 1993.

10. Audouin J, Comperat E, Le Tourneau A, Camilleri-Broët S, Adida C, Molina T and Diebold J: Myeloid sarcoma: Clinical and morphologic criteria useful for diagnosis. Int J Surg Pathol 11: 271-282, 2003

11. Amador-Ortiz C, Hurley MY, Ghahramani GK, Frisch S, Klco JM, Lind AC, Nguyen TT, Hassan A, Kreisel FH and Frater JL: Use of classic and novel immunohistochemical markers in the diagnosis of cutaneous myeloid sarcoma. J Cutan Pathol 38: 945-953, 2011.
12. Xavier SG, Fagundes EM, Hassan R, Bacchi C, Conchon M, Tabak DG, Spector N and Zalcberg IR: Granulocytic sarcoma of the small intestine with CBFbeta/MYH11 fusion gene: Report of an aleukaemic case and review of the literature. Leuk Res 27: 1063-1066, 2003.

13. Skalska-Sadowska J, Januszkiewicz-Lewandowska D, Derwich K, Pieczonka A, Samborska M and Wachowiak J: Ph-negative isolated myeloid sarcoma with NPM1 gene mutation in adolescent with Ph-positive chronic myeloid leukemia in remission after treatment with allogeneic bone marrow transplantation and imatinib mesylate. Pediatr Blood Cancer 62: 1070-1071, 2015.

14. Deeb G, Baer MR, Gaile DP, Sait SN, Barcos M, Wetzler M, Conroy JM, Nowak NJ, Cowell JK and Cheney RT: Genomic profiling of myeloid sarcoma by array comparative genomic hybridization. Genes Chromosomes Cancer 44: 373-383, 2005.

15. Menasce LP, Banerjee SS, Beckett E and Harris M: Extramedullary myeloid tumour (granulocytic sarcoma) is often misdiagnosed: A study of 26 cases. Histopathology 34: 391-398, 1999.

16. Movassaghian M, Brunner AM, Blonquist TM, Sadrzadeh H, Bhatia A, Perry AM, Attar EC, Amrein PC, Ballen KK, Neuberg DS and Fathi AT: Presentation and outcomes among patients with isolated myeloid sarcoma: A surveillance, epidemiology and end results database analysis. Leuk Lymphoma 56: 1698-1703, 2015.

17. Yamauchi $\mathrm{K}$ and Yasuda $\mathrm{M}$ : Comparison in treatments of nonleukemic granulocytic sarcoma: Report of two cases and a review of 72 cases in the literature. Cancer 94: 1739-1746, 2002.

18. He J, Zhu L, Ye X, Li L, Zhu J, Zhang J, Xie W, Shi J, Zheng W, Wei G, et al: Clinical characteristics and prognosis of nonleukemic myeloid sarcoma. Am J Med Sci 347: 434-438, 2014.

19. Chelly I, Mekni A, Kchir N, Karim BH, Khadija B, Selma B, Slim H, Khaldi M and Zitouna M: Intracerebellar granulocytic sarcoma. A case report. Pathologica 97: 335-337, 2005.

20. Tsimberidou AM, Kantarjian HM, Wen S, Keating MJ, O'Brien S, Brandt M, Pierce S, Freireich EJ, Medeiros LJ and Estey E: Myeloid sarcoma is associated with superior event-free survival and overall survival compared with acute myeloid leukemia. Cancer 113: 1370-1378, 2008.

21. Widhalm G, Dietrich W, Müllauer L, Streubel B, Rabitsch W, Kotter MR, Knosp E and Roessler K: Myeloid sarcoma with multiple lesions of the central nervous system in a patient without leukemia. Case report. J Neurosurg 105: 916-919, 2006.

22. Chevallier P, Mohty M, Lioure B, Michel G, Contentin N, Deconinck E, Bordigoni P, Vernant JP, Hunault M, Vigouroux S, et al: Allogeneic hematopoietic stem-cell transplantation for myeloid sarcoma: A retrospective study from the SFGM-TC. J Clin Oncol 26: 4940-4943, 2008.

23. Yagi T, Ishikawa J, Takahashi M, Yamashita Y, Kusakabe S, Yoshinami T, Masaie H, Sugimoto N, Yoshida H and Imamura F: Successful treatment of duodenal myeloid sarcoma with allogeneic bone marrow transplantation and additional radiotherapy. Intern Med 51: 769-772, 2012. 